\title{
Ultrastructural study of hippocampal cortex neurons in an experimental model of valproate encephalopathy
}

\author{
Krzysztof Sendrowski $^{1}$, Wojciech Sobaniec ${ }^{1}$, Piotr Sobaniec ${ }^{1}$, Maria E. Sobaniec-Lotowska ${ }^{2}$ \\ ${ }^{1}$ Department of Pediatric Neurology and Rehabilitation, Medical University of Bialystok, Poland \\ ${ }^{2}$ Department of Medical Pathomorphology, Medical University of Bialystok, Poland
}

\begin{abstract}
Valproate (VPA) is a widely used antiepileptic drug. A serious neurological-outcome defined as valproate encephalopathy (VE) may rarely occur during VPA therapy. Structural abnormalities within neurons are postulated as one of the reasons for VE. The aim of this study was to assess the ultrastructure of neurons in the hippocampal cortex during the course of chronic application of VPA to rats. VPA was chronically administered to rats, intragastrically, once daily at a dose of $200 \mathrm{mg} / \mathrm{kg}$ b.w. for $1,3,6,9$ and 12 months. The samples of hippocampal cortex, after routine laboratory preparation, were examined by electron microscopy. The drug induced pronounced ultrastructural changes in the population of pyramidal neurons within the hippocampal cortex after 9 and 12 months of VPA administration. The most expressed abnormalities were observed within the mitochondria and manifested by fragmentation of crests and almost complete disappearance of intramitochondrial granules. Mitochondria of numerous neurons resembled large vacuolar structures. Widening, shortening and irregular distribution of rough endoplasmic reticulum was also found. A characteristic feature of damaged neurocytes in the last two phases of the experiment was the disintegration of nuclear chromatin and the presence of numerous lipofuscin deposits within hyaloplasm. These cells assumed the look of "dark neurons" and presented the ultrastructural features of apoptosis and necrosis. Our results indicate that long-term VPA administration to rats leads to aponecrosis of hippocampal neurons. (Folia Histochemica et Cytobiologica 2013, Vol. 51, No. 1, 31-37)
\end{abstract}

Key words: valproate encephalopathy, hippocampus, ultrastructure, neurons, mitochondria, RER, aponecrosis

\section{Introduction}

Valproic acid (VPA, 2-propylvaleric acid) and its derivate, valproate, are commonly used antiepileptic drugs with a high effectiveness in seizure control [1]. Additionally, VPA is widely used as a mood-stabilizer in bipolar disorder [2], in the treatment of schizophrenia [3], neuropathic pain [4] as well as migraine headache prophylaxis [5]. Due to its beneficial activity in reducing the incidence of both generalized and partial seizures in adults and children, the drug belongs to the so called "first-line" anticonvulsive agents

Correspondence address: K. Sendrowski, Department of Pediatric Neurology and Rehabilitation, Medical University of Bialystok; Waszyngtona St. 17; 15-274 Bialystok, Poland, tel./fax: +48 8574508 12;

e-mail: krsen@wp.pl and belongs to the most often prescribed antiepileptic drugs. VPA is generally considered a safe and welltolerated drug, especially when compared with older antiepileptic agents such as phenobarbital and phenytoin. Adverse effects associated with the drug are usually mild and transient. Gastrointestinal disturbances (nausea, dyspepsia), weight gain, tremor, hair loss and elevation of liver enzyme activity are commonly reported $[1,6]$. On the other hand, rare but serious complications such as encephalopathy [7], parkinsonism and a progressive dementia-like syndrome $[8$, 9] may occur in the course of VPA-treatment.

Valproate encephalopathy (VE) is a serious neurological-outcome associated with long-term VPA application. In contrast to VPA intoxication, which is closely connected with a toxic concentration of the drug in blood serum, VE can occur even if VPA serum-concentration is within a therapeutic range [10]. 
The clinical picture of VE is complex and shares elements of various neurological symptoms, originating mainly from cerebellum (ataxia, nystagmus), the extrapyramidal system (tremor, parkinsonism) and hippocampus (memory disturbances, dementia-like syndrome). The exact pathomechanism of VE still remains not fully elucidated. VE is frequently accompanied by hyperammonemia without signs of liver failure. Hyperammonemia has been regarded as one of the most important causative factors of VE [7, 11]. However, in many patients with clinical features of $\mathrm{VE}$, the ammonia serum level was only slightly elevated or normal $[12,13]$. Thus, elevated serum ammonia cannot fully explain the encephalopathic effect of VPA. Based on these observations, VE might be rather of multi-factorial origin than only secondary to hyperammonemia. The adverse effects of VPA, including VE, have been investigated carefully by neuropharmacologists, however, neuropathologic investigations, are extremely scarce. Therefore, the basic aim of this study was to assess the ultrastructure of neurons in the cortex of the hippocampal gyrus during the course of chronic application of VPA. This study is a continuation of earlier investigations performed in our research center. It supplements our previously reported ultrastructural investigations of the other brain regions in the same model of chronic experimental VE [14-18].

\section{Material and methods}

Two groups of three-month-old male Wistar rats of an initial body mass of 160-180 g, preselected according to standard pharmacological screening tests, were used in the experiment. The animals were kept in a well-lit room at 18 $-20^{\circ} \mathrm{C}$ and fed standard granulated rat chow and tap water. All procedures were carried out in strict accordance with the Helsinki Convention guidelines for the care and use of laboratory animals. The study was approved by the Ethical Committee of the Medical University of Bialystok.

Group I consisted of 30 rats receiving sodium valproate (Vupral, Polfa) once daily in a fasting state through an intragastric tube, at the effective dose of $200 \mathrm{mg} / \mathrm{kg}$ b.w. for 1 , 3, 6, 9 and 12 months (six animals in each subgroup).

Group II contained 10 control animals matched in respect to age with the experimental animals, receiving physiological saline in the same way as the group I rats treated with VPA. All rats were weighed every two weeks to verify the effectiveness of VPA in the group I. Serum concentrations of VPA in group I were measured by gas chromatography and ranged between 60 and $135 \mu \mathrm{g} / \mathrm{mL}$ (mean 111.33 $\mu \mathrm{g} / \mathrm{mL}$; SD 21.61).

In the final stage of the experiment, $24 \mathrm{~h}$ after the termination of VPA administration, the animals were sacri- ficed under Nembutal anesthesia using a dose of $25 \mathrm{mg} / \mathrm{kg}$ b.w. by intravital intracardiac perfusion with $2.5 \%$ glutaraldehyde in $0.1 \mathrm{M}$ cacodylate buffer, $\mathrm{pH} 7.4$, at a constant pressure of $80 \mathrm{mmHg}$. Small tissue blocks ( $1 \mathrm{~mm}^{3}$ volume) were taken from the gyrus hippocampal cortex (using a magnifying glass), fixed in $3.6 \%$ glutaraldehyde in $0.1 \mathrm{M}$ cacodylate buffer ( $\mathrm{pH} 7.4$ ) for $2.5 \mathrm{~h}$ and washed in the same buffer for $18 \mathrm{~h}$. They were then postfixed in $2 \%$ osmium tetroxide in $0.1 \mathrm{M}$ cocodylate buffer ( $\mathrm{pH}$ 7.4) for $1 \mathrm{~h}$. Subsequently, the material, after dehydration in ethanol and propylene oxide, was embedded in Epon 812. Ultra-thin sections were double contrasted with $2 \%$ uranyl acetate and lead citrate, and examined under an OPTON 900 PC transmission electron microscope (Zeiss, Oberkochen, Germany) $[14,15]$. The material obtained from the hippocampal cortex of the control group was processed using the same techniques as for the VPA-receiving rats.

\section{Results}

The first ultrastructural abnormalities within the population of hippocampal neurons were observed after 6 months of valproate administration. In this group, perikarya of only few neurons were slightly or moderately enlarged and swollen. Structural abnormalities related to the mitochondria and granular (rough) endoplasmic reticulum (RER). Some mitochondria were enlarged and showed rarefaction of the matrix and partial loss of cristae. Quite frequently, the granular endoplasmic reticulum of the perikarya had irregularly arranged channels, which were dilated and shortened (Figure 1).

After 9 and 12 months of VPA administration prominent ultrastructural changes were observed in the perikarya of hippocampal neurons as well as their dendritic processes. Usually, pathological changes within the perikarya were more prominently expressed than in dendrites. Two types of neuronal injury were found. The cells were either enlarged and swollen or contracted with cytoplasm showing increased electron density. The ultrastructure of these cells corresponded with aponecrotic dark neurons. Sometimes, both types of neurons adhered to one another (Figure 2).

Electron microscopic examination of the hippocampal neurons revealed the most prominent abnormalities within mitochondria. The mitochondria of the damaged neurons exhibited features of regressive changes of differing severity. Often, these organelles would group in the peripheral areas of the hyaloplasm (Figures 2,3). Mitochondrial damage was manifested by fragmentation of crests, including their breakdown, as well as almost complete disappearance of intramitochondrial granules. Sometimes mitochondria resembled large electron-translucent vacuoles 


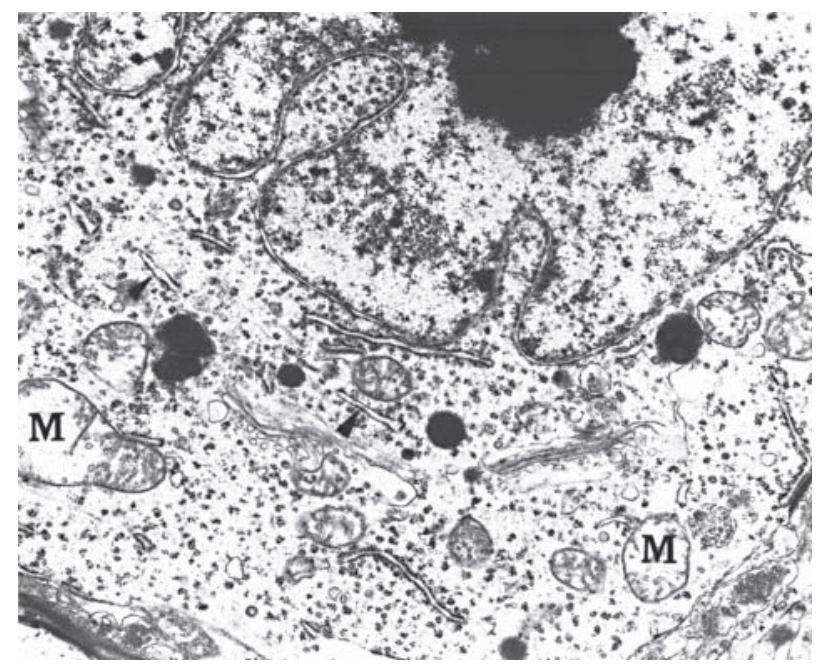

Figure 1. Perinuclear zone of the pyramidal neuron in hippocampus. Nuclear membrane with deep protrusion toward nucleoplasm, large nucleolus. Some swollen mitochondria (M) of a deserted matrix with focal breakdown of the mitochondrial crests, shortened channels of granular endoplasmic reticulum $(>)$. After 6 months of VPA administration. Original magnification $7000 \times$

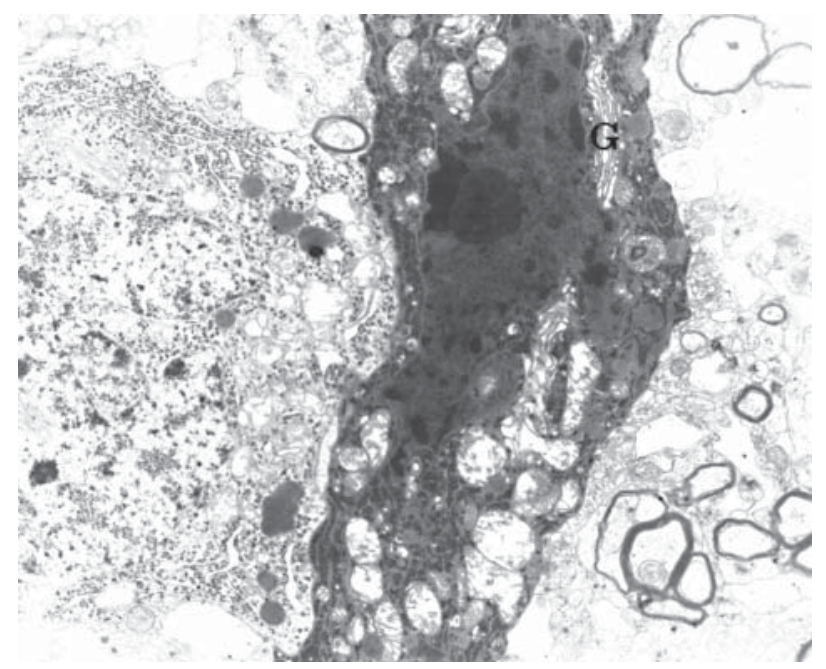

Figure 2. Adjacent perikarya of two neurons. One appears as a dark neuron and shows marked disintegration of karyoplasm and cytoplasm; cluster of swollen mitochondria inside the cytoplasm; G-Golgi apparatus. The other neuron's perikaryon (on the left) is swollen; intracellular organelles are quite well preserved. Lipofuscin deposits are visible. 9 months of VPA administration. $4400 \times$

(Figures 3, 4). It should be noted that pathological features were not found in all mitochondria, since some preserved normal ultrastructure (Figure 5).

A high degree of abnormalities were also observed within the granular endoplasmic reticulum. In com-

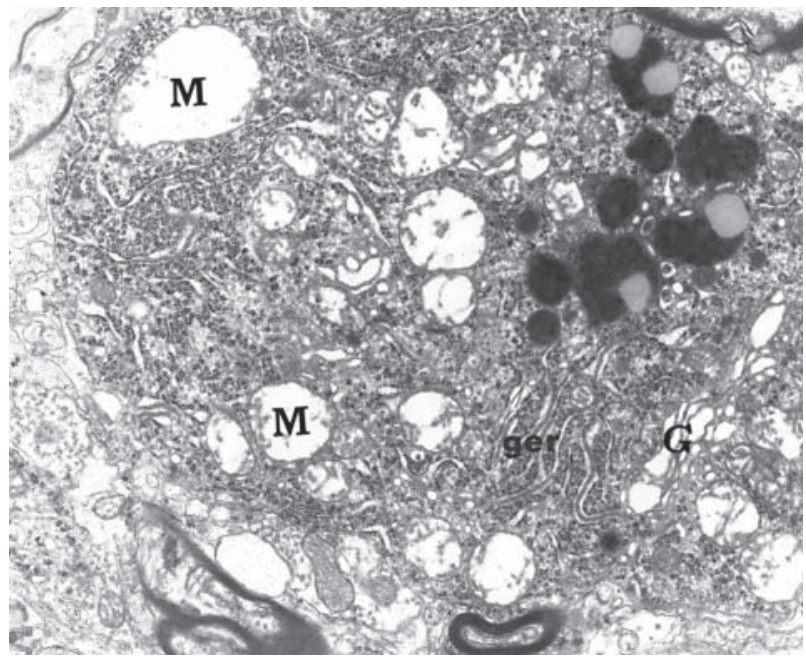

Figure 3. Fragment of perikarya of pyramidal neuron containing massively swollen mitochondria (M) and large cluster of lipofuscin granules. Channels of granular endoplasmic reticulum (ger) with a fairly well-preserved structure are chaotically scattered throughout the cytoplasm. Numerous polysomes and free ribosomes, G - Golgi. After 9 months of VPA administration. $7000 \times$

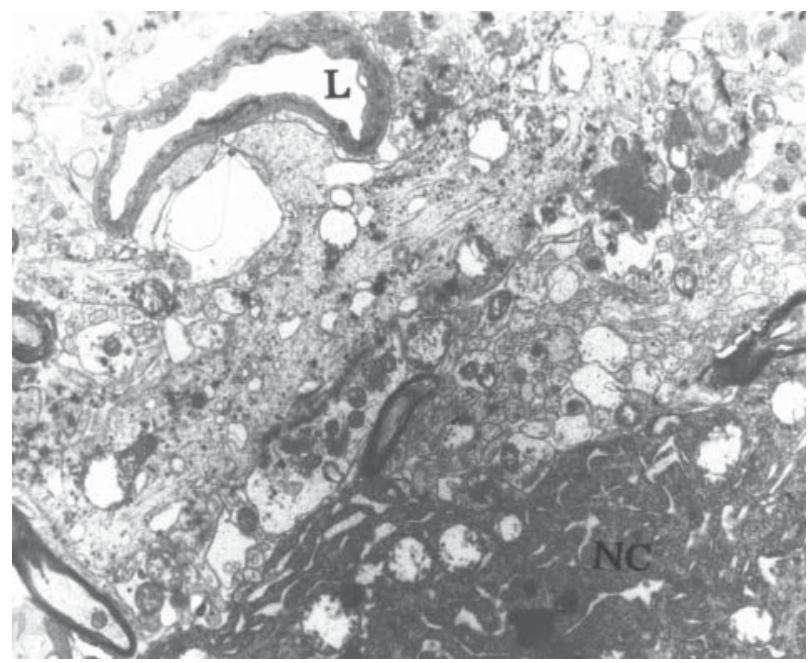

Figure 4. Fragment of perikarya of pyramidal nerve cell (NC) with increased electron density of cytoplasm, containing numerous changed mitochondria and scattered small lipid deposits. In the upper part capillary with a narrowed lumen $(\mathrm{L})$ is present. After 9 months of VPA administration. $3000 \times$

parison with rats subjected to shorter periods of VPA exposure, which neurons contained reticulum channels forming regular parallel formations, in rats with long exposure to VPA the RER channels were usually characterized by a significant widening, shortening 


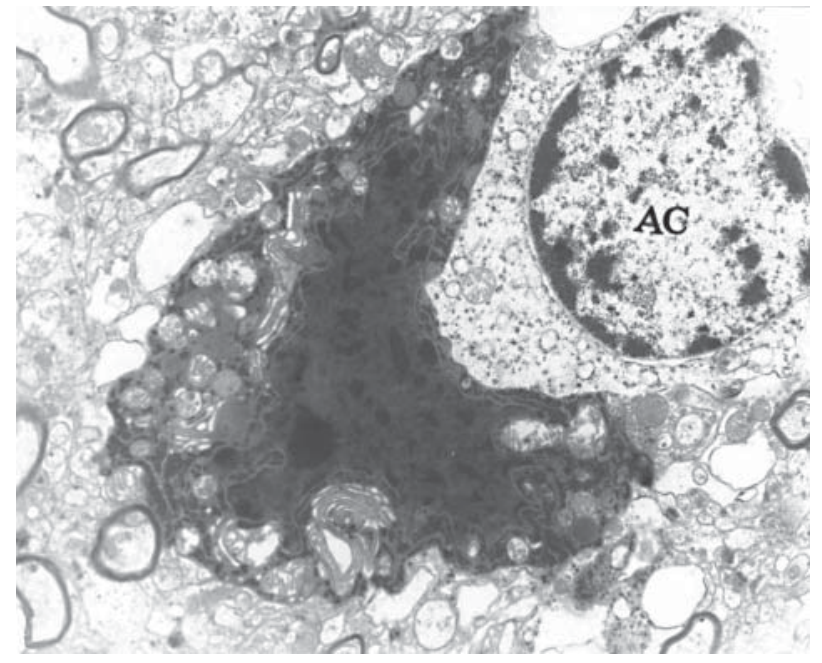

Figure 5. Dark, shrunk, aponecrotic neuron with total disintegration of karyoplasm. Mitochondria cells are fairly well preserved. Protoplasmatic astrocyte (AC) adjacent to a neuron. After 12 months of VPA administration. $4400 \times$

and irregular distribution in different areas of the hyaloplasm (Figure 3). Most pronounced abnormalities of granular endoplasmic reticulum were observed in the „dark neurons”. Inside these neurons RER exhibited complete degeneration, and their place was filled with dark microgranular, sometimes homogeneous material. The Golgi body in this group of experimental animals was characterized by a considerable degree of expansion, which was significant both for the dark, shrunk neurons, as well as those with features of swelling. Golgi was visible in the form of elongated and widened, arranged in several rows of tubular connections and cisternae with vesicles located at the periphery. Extensive structures of the Golgi apparatus were spread throughout the area of the hyaloplasm, also close to plasma membrane. „Dark neurons" were characterized by significant disintegration and degeneration of nuclei and endoplasmic structures (Figures 2, 5, 6, 7).

The nuclei of these neurocytes were shrunk with dense, granular nucleoplasma. Areas devoid of organelles, filled with homogeneous dark microgranular or homogeneous material, were also found in hyaloplasm. A characteristic feature of neurocytes which demonstrated both types of damage was the presence within their hyaloplasm of numerous lipofuscin granules (Figures 2, 3, 4).

Ultrastructure of the hippocampal neurons in agematched control rats (treated with vehicle) was normal at each stage of the experiment (Figure 8).

After 9 and 12 months of the experiment, single lipofuscin granules within the hyaloplasm of a few neurons were found (Figure 9).

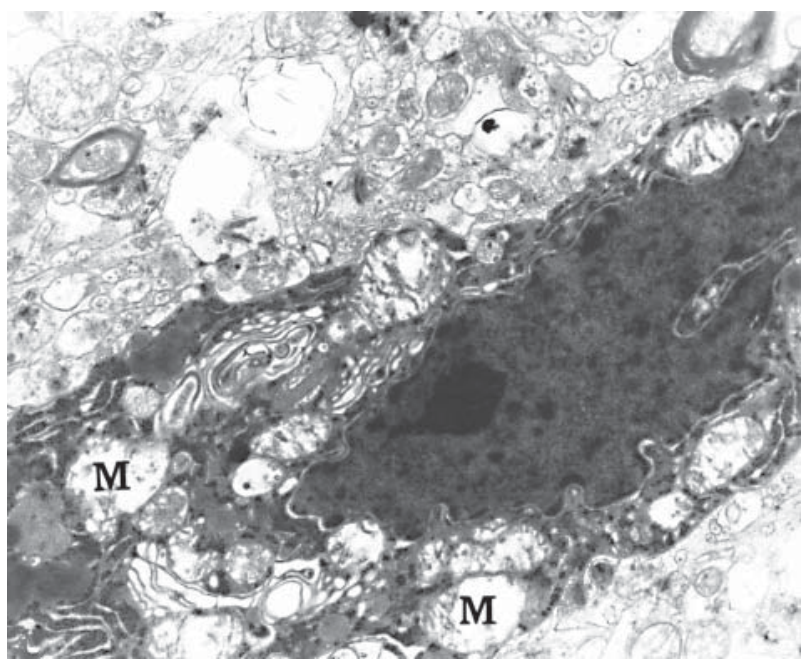

Figure 6. A fragment of dark, lethally damaged neuron. Visibly damaged mitochondria (M). After 12 months of VPA administration. $7000 \times$

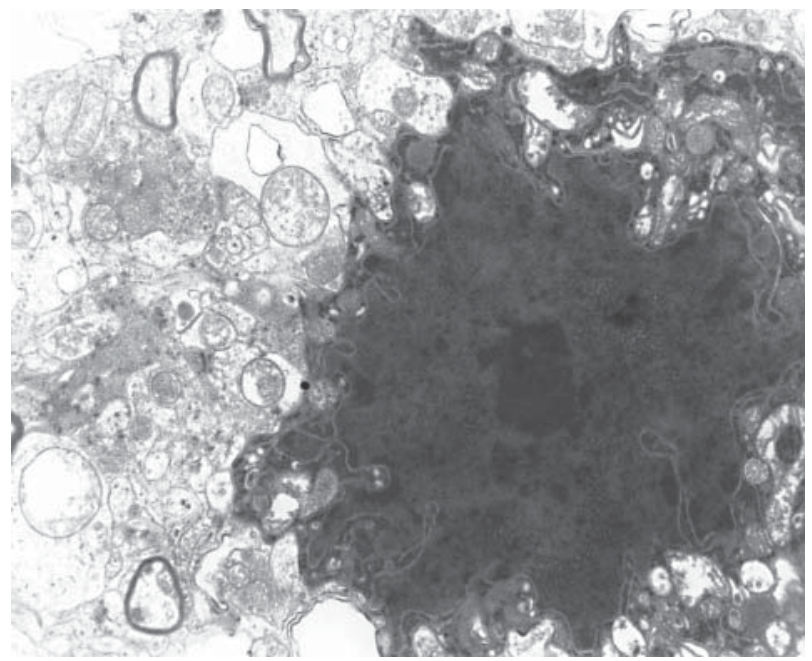

Figure 7. Image of a dark, shrunken neuron. After 12 months of VPA administration. $7000 \times$

\section{Discussion}

The results of experimental and clinical studies indicate that commonly used long-term antiepileptic drugs may cause several, sometimes very serious, adverse effects. It can be assumed that the basis of such states as drug-induced encephalopathy, including valproate encephalopathy, may be not only functional but also structural disorders of brain nerve tissue. In this paper, we presented the results of ultrastructural assessment of hippocampal neurons in a model of chronic valproate encephalopathy. The choice of 


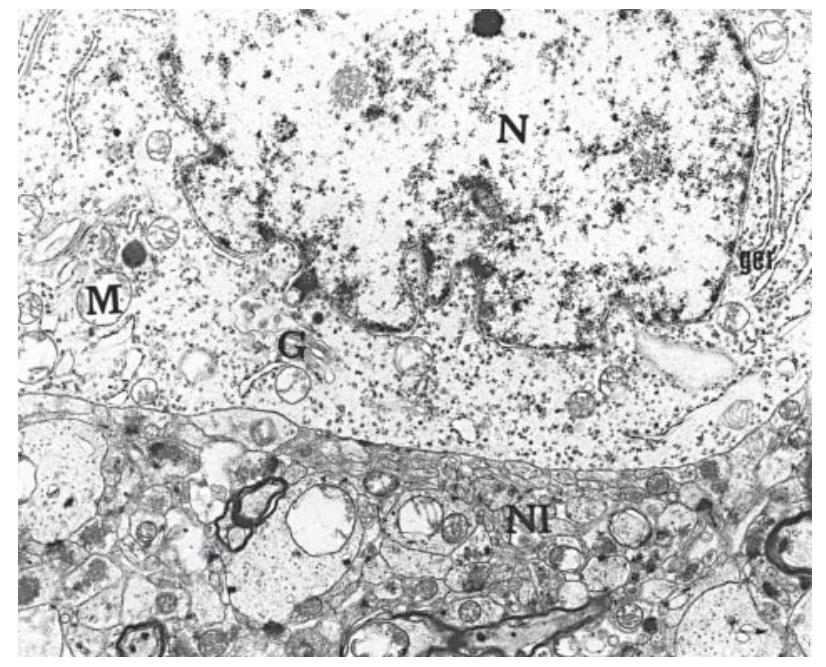

Figure 8. Normal ultrastructure of the perinuclear zone of the pyramidal neuron. Nucleus (N) with homogenously distributed euchromatin; small densities of heterochromatin. Well preserved endocellular organelles: mitochondria (M), granular endoplasmic reticulum (ger), Golgi apparatus (G); neuropil (NI). Control group (after 9 months of observation). $4400 \times$

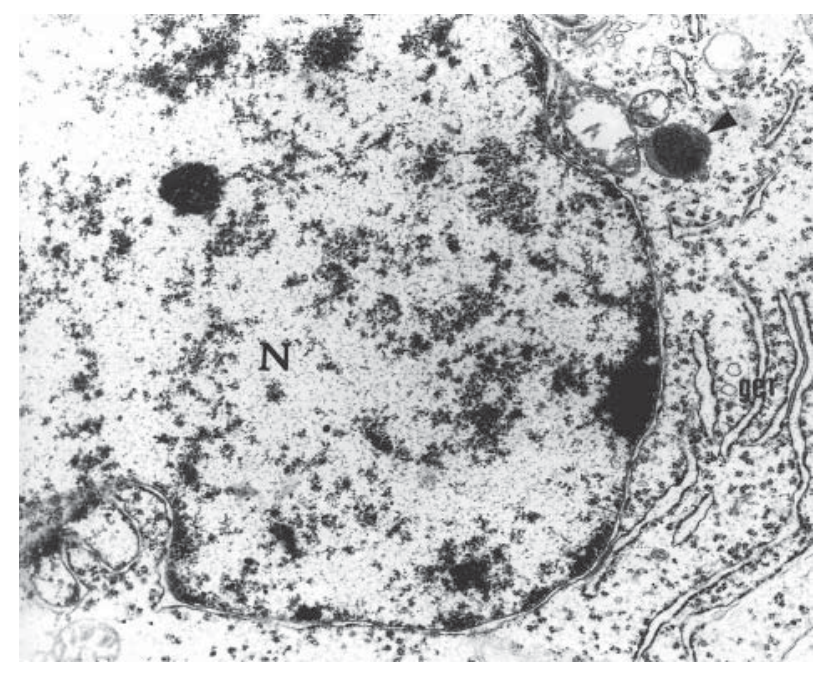

Figure 9. Perinuclear zone of the pyramidal neuron cytoplasm. N-cell nucleus containing homogenously distributed euchromatin and small clusters of heterochromatin; visible nucleolus. Regular, row arrangement of channels of the granular endoplasmic reticulum (ger), numerous ribosomes. A single lipofuscin granule $(>)$. Control group (after 12 months of observation); $7000 \times$

studying the ammonal cortex was not random. It is known that hippocampus has a specific sensitivity to both endogenous and exogenous damaging agents; furthermore in the archicortex structure, we observed the highest concentrations of the studied drug, sodi- um valproate and its metabolites, compared with other areas of the brain $[19,20]$. Therefore, we assumed that any neuropathological lesions will be found and will be the most likely expressed in the ammonal cortex. In a long-term experimental rat model intragastrically administered VPA for 1, 3, 6, 9 and 12 months at a dose of $200 \mathrm{mg} / \mathrm{kg}$ of body weight/day resulted in discrete pathological changes in the ultrastructure of hippocampal neurons after 6 months of the experiment, and significant changes only in the later phases of the experiment, that is after 9 and 12 months. The abnormalities found were mainly in endocellular organelles, and to a lesser extent in cell nuclei. With increasing duration of VPA administration we observed a gradual intensification of changes. While in the group of rats after 6 months of exposure to VPA changes were observed almost exclusively within mitochondria, in the subsequent phases of the experiment these anomalies not only intensified significantly, but were also accompanied by other, related to other cytoplasmic and karyoplasmic structures. Starting from the 9th month of the experiment, we found numerous nerve cells with pleated cell membrane, shrunk, containing the nucleus of a dark, uniformly condensed nucleoplasma with organelles expressing variously severe degrees of disintegration, including degeneration. Often such cells bordered with neurons of a normal ultrastructure. The above type of damage to the nerve cells of the hippocampal cortex, which was also characteristic for cortical cerebellar Purkinje cells in the same experimental model of VPA [14], corresponded to the image of the so-called 'dark neurons' described in the literature as typical for the ischemic processes of the central nervous system [21-23]. Recent electron-microscopic observations suggested that similar 'dark neurons' were found in traumatic [24], electric [24], chemical [25] and hypoglycemic [26] models of neuronal injury. These cells have the ultrastructural features of both apoptosis (disintegration and focal concentration of nuclear chromatin) and necrosis processes (severe damage to mitochondria, disintegration of granular endoplasmic reticulum, dilation of channels and cisterns of Golgi and accumulation of lipofuscin deposits). These observations suggest that the pathways of VPA-mediated damage to neurons are complex. It is generally accepted that death of a nerve cell can occur as a result of necrosis or apoptosis. Necrosis occurs in a short time and is the result of sudden intracellular change in ion concentrations, swelling of the cell and lysis. In contrast to necrosis, apoptosis involves a series of biochemical phenomena that lead to the destruction of genetic material and programmed cell death [27]. Recent studies have proved that both these 
phenomena usually occur simultaneously and are referred to as aponecrosis [28]. The mechanism of this process is extremely complex, because it combines the unfavorable interaction of calcium ions, excitatory amino acids, the products of lipid cell membrane degradation, oxygen free radicals and autoimmune response. An extremely important role in the pathogenesis of neuron death is played by mitochondria and endoplasmic reticulum, which are intracellular deposits of calcium ions. Oxygen free radicals together with calcium ions cause the opening of so-called megachannels in the mitochondrial membrane, which irreversibly destroys these organelles and interferes with the mechanisms of ATP generation [29]. These phenomena are also accompanied by the overexpression of pro-apoptotic genes $[30,31]$. $\mathrm{Ca}^{2+}$ and its movements into and out of the cell present an important mechanisms in the control of normal cell physiology [32]. Any dysfunction in the movement from outside to inside the cell or between organelles may have extremely negative effects, and the disturbance may lead to aponecrosis [33, 34]. In our opinion, disturbances in $\mathrm{Ca}^{2+}$ fluxes, pathological alterations of reduction/ /oxidation pathways, irreversible mitochondria damage followed by aponecrosis may contribute to the demonstrated VPA-mediated toxicity within hippocampal neurons.

\section{Conclusions}

The first, mildly-expressed ultrastructural changes within the hippocampal neurons were observed only after 6 months of VPA administration. After 9 and 12 months of VPA exposure, many neural cells of the hippocampal pyramidal layer were severely damaged. These cells assumed the look of dark neurons and presented the ultrastructural features of both apoptosis and necrosis processes. Thus, long-term VPA administration to rats leads to aponecrosis of hippocampal neurons.

\section{References}

1. Davis R, Peters DH, McTavish D. Valproic acid. A reappraisal of its pharmacological properties and clinical efficacy in epilepsy. Drugs.1994;47:332-372.

2. Kessing LV, Hellmund G, Geddes JR, Goodwin GM, Andersen PK. Valproate v. lithium in the treatment of bipolar disorder in clinical practice: observational nationwide register-based cohort study. Br J Psychiatry.2011;199:57-63.

3. Haddad PM, Das A, Ashfag M, Wieck A. A review of valproate in psychiatric practice. Expert Opin Drug Metab Toxicol. 2009;5:539-551.

4. Waszkielewicz AM, Gunia A, Słoczyńska K, Marona H. Evaluation of anticonvulsants for possible use in neuropathic pain. Curr Med Chem. 2011;18:4344-4358.

5. Vikelis M, Rapoport AM. Role of antiepileptic drugs as preventive agents for migraine. CNS Drugs. 2010;24:21-33.
6. Jankovic SM, Dostic M. Choice of antiepileptic drugs for the elderly: possible drug interactions and adverse effects. Expert Opin Drug Metab Toxicol. 2012;8:81-91.

7. Lewis C, Deshpande A, Tesar GE et al. Valproate-induced hyperammonemic-encephalopathy: a brief review. Curr Med Res Opin. 2012;28:1039-1042.

8. Ristic AJ, Vojvodic N, Jankovic S, Sindelic A, Sokic D. The frequency of reversible parkinsonism and cognitive decline associated with valproate treatment: a study of 364 patients with different types of epilepsy. Epilepsia. 2006;47:2183-2185.

9. Masmoudi K, Gras-Champel V, Bonnet I et al. Dementia and extrapyramidal problems caused by long-term valproic acid. Therapie. 2000;55:629-634. In French.

10. Rousseau MC, Montana M, Villano P et al. Valproic acidinduced encephalopathy in very long course treated patients. Brain Inj. 2009;23:981-984.

11. Chopra A, Kolla BP, Mansukhani MP, Netzel P, Frye MA. Valproate-induced hyperammonemicencephalopathy: an update on risk factors, clinical correlates and management. Gen Hosp Psychiatry. 2012;34:290-298.

12. Gerstner T, Buesing D, Longin E et al. Valproic acid induced encephalopathy-19 new cases in Germany from 1994 to 2003-a side effect associated to VPA-therapy not only in young children. Seizure. 2006;15:443-448.

13. Parize P, Beuret P, Fischer C. A valproate-induced encephalopathy without hyperammonemia. Ann Fr Anesth Reanim. 2007;26:1084-1085. In French.

14. Sobaniec-Łotowska ME. Ultrastructure of Purkinje cell perikarya and their dendritic processes in the rat cerebellar cortex in experimental encephalopathy induced by chronic application of valproate. Int J ExpPathol. 2001;82:337-348.

15. Sobaniec-Łotowska ME. Ultrastructure of synaptic junctions in the cerebellar cortex in experimental valproate encephalopathy and after terminating chronic application of the antiepileptic. Folia Neuropathol. 2002;40:87-96.

16. Sobaniec--Lotowska ME. Ultrastructure of astrocytes in the cortex of the hippocampal gyrus and in the neocortex of the temporal lobe in experimental valproate encephalopathy and after valproate withdrawal. IntJ ExpPathol. 2003;84:115-125.

17. Sobaniec-Łotowska ME, Lotowska JM. Ultrastructural study of cerebellar dentate nucleus astrocytes in chronic experimental model with valproate. Folia Neuropathol. 2005;43: 166-171.

18. Sobaniec-Łotowska M, Sobaniec W, Augustynowicz A. Morphometric analysis of the cerebellar cortex capillaries in the course of experimental valproate encephalopathy and after chronic exposure to sodium valproate using transmission electron microscopy. Folia Neuropathol. 2001; 39:277-280.

19. Semmes RL, Shen DD. Comparative pharmacodynamics and brain distribution of E-delta 2-valproate and valproate in rats. Epilepsia. 1991;32:232-241.

20. Adkison KD, Ojemann GA, Rapport RL, Dills RL, Shen DD. Distribution of unsaturated metabolites of valproate in human and rat brain - pharmacologic relevance? Epilepsia. 1995;36:772-782.

21. Brierley JB, Meldrum BS, Brown AW. The threshold and neuropathology of cerebral ,anoxic-ischemic” cell change. Arch Neurol. 1973;29:367-374.

22. Mossakowski MJ, Gajkowska B, Tsitsishvili A. Ultrastructure of neurons from the CA1 sector of Ammon's horn in shortterm cerebral ischemia in Mongolian gerbils. Neuropatol Pol. 1989;27:39-53.

23. Matyja E, Kida E. Hippocampal damage in vitro after different periods of oxygen deprivation. Neuropatol Pol. 1992;30: 231-243. 
24. Csordás A, Mázló M, Gallyas F. Recovery versus death of "dark" (compacted) neurons in non-impaired parenchymal environment. Acta Neuropathol. 2003;106: 37-49.

25. Baracskay P, Szepesi Z, Orbán G, Juhász G, Czurkó A. Generalization of seizures parallels the formation of „dark” neurons in the hippocampus and pontine reticular formation after focal-cortical application of 4-aminopyridine (4-AP) in the rat. Brain Res. 2008;1228:217-228.

26. Gallyas F, Csordás A, Schwarcz A, Mázló M."Dark" (compacted) neurons may not die through the necrotic pathway. Exp Brain Res. 2005;160:473-486.

27. Yakovlev AG, Faden AI. Mechanisms of neural cell death: implications for development of neuroprotective treatment strategies. Neuro Rx. 2004;1:5-16.

28. Formigli L, Papucci L, Tani A et al. Aponecrosis: morphological and biochemical exploration of a syncretic process of cell death sharing apoptosis and necrosis. J Cell Physiol. 2000;182:41-49.
29. Marzo I, Brenner C, Kroemer G. The central role of the mitochondrial megachannel in apoptosis: evidence obtained with intact cells, isolated mitochondria, and purified protein complexes. Biomed Pharmacother. 1998;52:248-251.

30. Szkudlarek M, Lebelt A, Klim B et al. Apoptosis phenomenon in the selected neoplasms of the glial origin. Rocz Akad Med. Bialymst. 2004;49:16-18.

31. Jin H, Kanthasamy A, Anantharam V, Rana A, Kanthasamy AG.Transcriptional regulation of pro-apoptotic protein kinase Cdelta: implications for oxidative stress-induced neuronal cell death. J Biol Chem. 2011;286:19840-19859.

32. Bootman MD, Collins TJ, Peppiatt CM et al. Calcium signaling — an overview. Semin Cell Dev Biol. 2001;12:3-10.

33. Berliocchi L, Bano D, Nicotera P. Ca2+ signals and death programmes in neurons. Philos Trans R SocLondB Biol Sci. 2005;360:2255-2258

34. Pretorius E, Bornman MS. Calcium-mediated aponecrosis plays a central role in the pathogenesis of estrogenic chemical-induced neurotoxicity. Med Hypoth. 2005;65:893-904.

Submitted: 6 October, 2012

Accepted after reviews: 8 February, 2013 